\title{
Impacts of subsynchronous and supersynchronous frequency components on synchrophasor measurements
}

\author{
Hao LIU ${ }^{1}$, Tianshu BI ${ }^{1}$, Xiqiang CHANG ${ }^{2}$, Xiaolong GUO ${ }^{2}$, \\ Lu WANG ${ }^{1}$, Chuang CAO ${ }^{1}$, Qing YAN $^{2}$, Jinsong LI $^{3}$
}

\begin{abstract}
Phasor measurement units (PMU) are playing an increasingly important role in power system dynamic security monitoring and control. However, the wide-area deployments of the renewable energy sources and the high voltage direct current (HVDC) transmission bring a large number of inter-harmonics to the power grid, which may result in further power system security problems. The impacts of inter-harmonics on synchrophasor measurements are revealed. This paper derives the phasor expressions of the signal, which contains the fundamental component and the inter-harmonics. It is found that the inter-harmonics will lead to the subsynchronous oscillation of the phasor measurements. The frequency transmutation principle between the harmonic and the phasor oscillation is revealed. Then, the field PMU data recorded during a subsynchronous oscillation, which occurred in an area of China with a high concentration of wind farms and HVDC transmission lines, are studied. A geographical wiring diagram with the subsynchronous oscillation distribution
\end{abstract}

CrossCheck date: 1 June 2016

Received: 30 November 2015/Accepted: 5 June 2016/Published online: 22 July 2016

(C) The Author(s) 2016. This article is published with open access at Springerlink.com

$\triangle$ Tianshu BI

tsbi@ncepu.edu.cn

Hao LIU

hliu@ncepu.edu.cn

Xiqiang CHANG

changxiqiang@xj.sgcc.com.cn

Xiaolong GUO

guoxiaolong@xj.sgcc.com.cn

Lu WANG

595459832@qq.com depicts the severe consequences of the inter-harmonics. In addition, the correctness of the theoretical derivation and the possibility of the inter-harmonics monitoring are verified.

Keywords Inter-harmonics, Frequency, Subsynchronous oscillations, Phasor measurement units (PMU), Renewable energy source

\section{Introduction}

In recent years, the wide implementation of phasor measurement units (PMU) results in the increasing developments of the power system dynamic security monitoring and control based on PMU [1-4], such as the post-event analysis [5], the low-frequency oscillation monitoring [6], the model validation, the parameter identification [5], and so on. The synchronized, fast and accurate phasor measurements of the fundamental component obtained by

\section{Chuang CAO}

1149161748@qq.com

Qing YAN

yanqing@xj.sgcc.com.cn

Jinsong LI

lijinsong@epri.sgcc.com.cn

1 State Key Laboratory of Alternate Electrical Power System with Renewable Energy Sources, North China Electric Power University, Beijing 102206, China

2 State Grid Xinjiang Electric Power Company, Xinjiang 830063, China

3 Power Automation Department, China Electric Power Research Institute, Beijing 100192, China 
PMUs have brought about a revolution in the dynamic security monitoring and control.

However, with the increasing development of the renewable energy and the high voltage direct current (HVDC) transmission, more and more electronic devices are deployed in the power grid. This introduces a large number of inter-harmonics that are not integer multiples of the fundamental component to the power signals, which changes the measurement of the fundamental component and may further threaten the operating security of the power system.

One of threatens is the subsynchronous oscillation, which is of great harm to the power system. Several subsynchronous oscillation events in the wind-integrated system are reported around the world [7], such as the Electric Reliability Council of Texas (ERCOT) in 2009 [8], Oklahoma Gas and Electric Company (OG\&E) in 2011 [9] and China Jibei Power Grid in 2013 and 2014 [10]. From the second half of the year 2015, there have been several subsynchronous oscillations caused by the inter-harmonics happened in a certain area of China with a high concentration of wind farms and HVDC transmission lines. Several thermal power generations nearby were tripped by their torsional stress relays (TSR), the power of more than $1 \mathrm{MW}$ was lost. Then, the wind farms were tripped off as well. The affected area was more than $130000 \mathrm{~km}^{2}$. The inter-harmonics included subsynchronous and supersynchronous ones, whose frequency range was wide, from 10 to $100 \mathrm{~Hz}$. Currently implemented harmonic monitoring devices are not suitable.

The discrete Fourier transform (DFT) is widely used for phasor estimation in PMUs [2]. But, DFT cannot extract the phasor precisely under dynamic conditions. With the release of the PMU standards around the world [11-14], many technologies have been developed to enhance the performance of PMUs [15]. A DFT-based algorithm is developed in [16] to obtain phasors when there are direct current (DC) components in the inputs. The Taylor series-based algorithm is carried out in [17] to improve the performance of PMU under dynamic conditions. Nevertheless, none of the references deals with exploring the phasor measurement of signals with interharmonics.

In this paper, the phasor expressions of the signals, which contain the fundamental component and the interharmonics, are derived. It is found that both subsynchronous and supersynchronous harmonic will result in the subsynchronous oscillation in phasor measurements. The mechanism frequency transmutation between the inter-harmonic and the phasor oscillation is revealed. Then, the recording data and the phasor measurements obtained by PMUs during the subsynchronous oscillation in the area of China with a high concentration of wind farms and HVDC transmission lines are investigated. The analysis results demonstrate the severe consequences of the inter-harmonics and verify the correctness of the theoretical derivation.

\section{Phasor expression of signal containing single inter-harmonic}

Under dynamic conditions, the pure stationary sinusoidal formula is not suitable even for an envelope of the signal within one calculation data window. The correct dynamic fundamental signal is expressed as:

$x(t)=\sqrt{2} X_{\mathrm{m}}(t) \cos \left(2 \pi f_{0} t+\left(2 \pi \int_{0}^{t} \Delta f(t) \mathrm{d} t+\varphi_{0}\right)\right)$

where $x(t)$ is the instantaneous value of the signal; $X_{\mathrm{m}}(t)$ the magnitude; $\varphi_{0}$ the initial phase angle; $f_{0}$ the nominal frequency; $\Delta f(t)$ the frequency deviation. The magnitude and the frequency of the signal are the functions with respect to time.

Then (1) is represented by a complex number $\dot{X}$ known as its dynamic phasor representation:

$$
\begin{aligned}
x(t) \leftrightarrow \dot{X} & =X_{\mathrm{m}}(t) \mathrm{e}^{\mathrm{j}\left(2 \pi \int_{0}^{t} \Delta f(t) \mathrm{d} t+\varphi_{0}\right)} \\
= & X_{\mathrm{m}}(t) \angle\left(2 \pi \int_{0}^{t} \Delta f(t) \mathrm{d} t+\varphi_{0}\right) \\
= & X_{\mathrm{m}}(t)\left(\cos \left(2 \pi \int_{0}^{t} \Delta f(t) \mathrm{d} t+\varphi_{0}\right)\right. \\
& \left.+\mathrm{j} \sin \left(2 \pi \int_{0}^{t} \Delta f(t) \mathrm{d} t+\varphi_{0}\right)\right)
\end{aligned}
$$

It is customary to suppress the term $\mathrm{e}^{\mathrm{j}\left(2 \pi f_{0} t\right)}$ in the expression above, with the understanding that the reference coordinate rotates with the angular frequency of $2 \pi f_{0}$. Therefore, the measured frequency by using the rotating coordinate is the difference between real frequency of the power system and the rated one. The further frequency measurement transformation is needed to obtain the real frequency of the power system.

Suppose the signal is

$x(t)=\sqrt{2} X_{\mathrm{m}} \cos \left(2 \pi f_{1} t+\varphi_{0}\right)$

where $f_{1}$ is the signal frequency.

Then, its phasor expression $\dot{X}$ is

$\dot{X}=X_{\mathrm{m}} \angle \varphi_{1}=X_{\mathrm{m}} \angle\left[2 \pi\left(f_{1}-f_{0}\right) t+\varphi_{0}\right]$

When the signal is the subsynchronous harmonic or supersynchronous one, $f_{1}$ need not be an integer multiple or divisor of $f_{0}$. As shown in (4), the phasor of the harmonic rotates with the speed of $2 \pi \Delta f_{1}=2 \pi\left(f_{1}-f_{0}\right)$. 
If the input contains the rated component and a harmonic component, the expression is shown as follows:

$$
x(t)=\sqrt{2} X_{\mathrm{m} 0} \cos \left(2 \pi f_{0} t+\varphi_{0}\right)+\sqrt{2} X_{\mathrm{m} 1} \cos \left(2 \pi f_{1} t+\varphi_{10}\right)
$$

where $X_{\mathrm{m} 0}$ is the magnitude of the rated component, $X_{\mathrm{m} 1}$ the magnitude of the harmonic component.

Then, its phasor expression $\dot{X}_{\mathrm{c} 1}$ is the superposition of the two components. In the real power system, all the phasors in this paper is expressed in a synchronously rotating coordinate. The relationship among three phasors is shown in the synchronously rotating coordinate (Fig. 1). It can be seen that $\dot{X}_{0}$ is static since it rotates with the coordinate at the same speed, while $\dot{X}_{1}$ rotates with the speed of $2 \pi \Delta f_{1}$. Therefore, $\dot{X}_{\mathrm{c} 1}$ rotates with the same speed. According to the cosine theorem, the formula of $\dot{X}_{\mathrm{c} 1}$ is given as:

$$
\left\{\begin{aligned}
\dot{X}_{\mathrm{c} 1}= & X_{\mathrm{mc} 1}-\varphi_{\mathrm{c} 1}=\dot{X}_{0}+\dot{X}_{1}= \\
& \sqrt{X_{\mathrm{m} 0}^{2}+X_{\mathrm{m} 1}^{2}+2 X_{\mathrm{m} 0} X_{\mathrm{m} 1} \cos \left(2 \pi \Delta f_{1} t\right)} \angle \beta \\
\beta= & \arctan \left(\frac{X_{\mathrm{m} 0} \sin \left(2 \pi \Delta f_{1} t\right)}{X_{\mathrm{m} 1}+X_{\mathrm{m} 1} \cos \left(2 \pi \Delta f_{1} t\right)}\right)
\end{aligned}\right.
$$

It can be observed that both the magnitude and the phase angle are the functions with respect to time.

Now suppose the input contains the harmonic whose $X_{\mathrm{m} 1}$ is $5.773 \mathrm{~V}$ and $f_{1}$ is $20 \mathrm{~Hz}$, and the rate signal whose $X_{\mathrm{m} 0}$ is $57.73 \mathrm{~V}$ and $f_{0}$ is $50 \mathrm{~Hz}$, then the magnitude and the phase angle of the superimposed phasor are outlined in Fig. 2. It can be observed that the superimposed phasor oscillates with the subsynchronous frequency of $\Delta f_{1}=30 \mathrm{~Hz}$. However, both of them are not pure but approximate sinusoidal curves, which can also be concluded from (6). That is also the reason why there are other frequency components in the magnitude and the phase angle spectrum, as shown in Fig. 2b, d. Therefore, the subsynchronous or supersynchronous harmonic will result in the subsynchronous oscillation of the superimposed phasor.

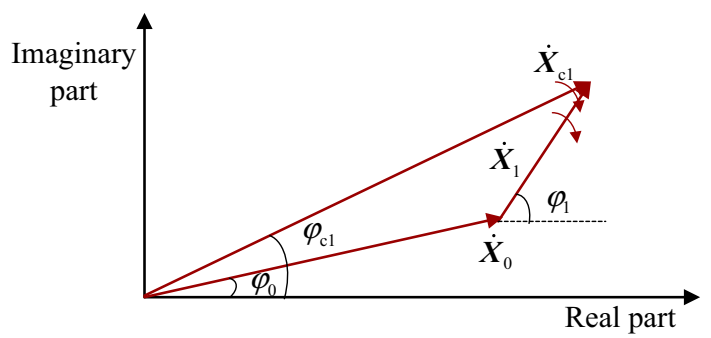

Fig. 1 Relationship of three phasors

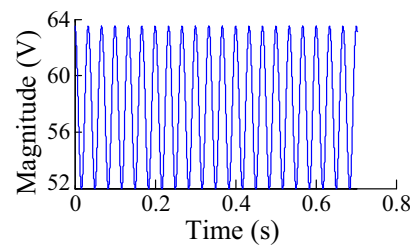

(a) Magnitude of phasor

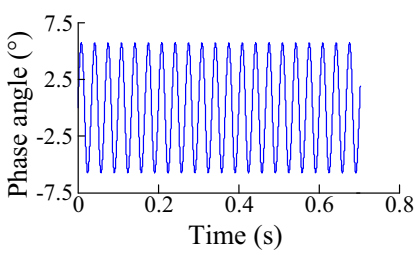

(c) Phase angle of phasor

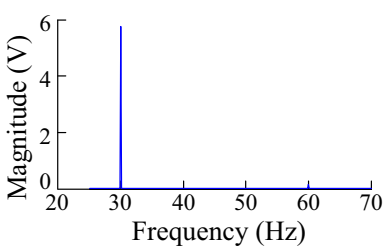

(b) Specturm of magnitude

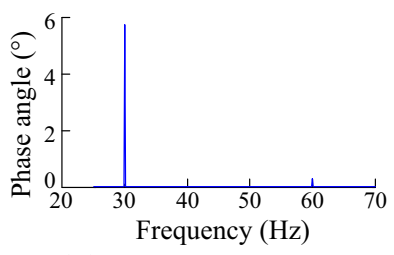

(d) Spectrum of phase angle
Fig. 2 Superimposed phasor with single harmonic

\section{Phasor expression containing multiple inter- harmonics}

In practice, several harmonics exist simultaneously in the transmission system. As an example, a signal with two harmonics is given as:

$$
\begin{aligned}
x(t)= & \sqrt{2} X_{\mathrm{m} 0} \cos \left(2 \pi f_{0} t\right)+\sqrt{2} X_{\mathrm{m} 1} \cos \left(2 \pi f_{1} t+\varphi_{10}\right) \\
& +\sqrt{2} X_{\mathrm{m} 2} \cos \left(2 \pi f_{2} t+\varphi_{20}\right)
\end{aligned}
$$

where $X_{\mathrm{m} 2}, f_{2}$ and $\varphi_{20}$ are the magnitude, the frequency and the initial phase angle of the second harmonic. To simplify the derivation process, $\varphi_{0}$ is set as $0^{\circ}$.

The superimposed phasor $\dot{X}_{\mathrm{c} 2}$ can be gotten by

$\dot{X}_{\mathrm{c} 2}=X_{\mathrm{mc} 2} \angle \varphi_{\mathrm{c} 2}=\dot{X}_{0}+\dot{X}_{1}+\dot{X}_{2}=\dot{X}_{\mathrm{c} 1}+\dot{X}_{2}$

where $X_{\mathrm{mc} 2}, \varphi_{\mathrm{c} 2}$ are the magnitude and the phase angle of $\dot{X}_{\mathrm{c} 2}$.

Since $\dot{X}_{1}$ and $\dot{X}_{2}$ keep rotating in the synchronously rotating coordinate, while $\dot{X}_{0}$ is static, the position of the three phasors keeps changing. Figure 3 shows six possible scenarios. It can be concluded that, since $\dot{X}_{\mathrm{c} 1}$ and $\varphi_{\mathrm{c} 2}$ can be calculated by (6), the key point to obtain $X_{\mathrm{mc} 2}$ and $\varphi_{\mathrm{c} 2}$ is obtaining $\varphi_{\mathrm{c} 2}^{\prime}$ and $\delta$ by the triangle formed by $\dot{X}_{\mathrm{c} 1}, \dot{X}_{2}$ and $\dot{X}_{\mathrm{c} 2}$, as shown in (9) and (10).

$X_{\mathrm{mc} 2}=\sqrt{X_{\mathrm{mc} 1}^{2}+X_{\mathrm{m} 2}^{2}-2 X_{\mathrm{mc} 1} X_{\mathrm{m} 2} \cos (\pi-\delta)}$

$\varphi_{\mathrm{c} 2}^{\prime}=\angle\left(\arctan \left(\frac{X_{\mathrm{m} 2} \sin \delta}{X_{\mathrm{mc} 1}+X_{\mathrm{m} 2} \cos \delta}\right)\right)$

However, the formulas of $\delta$ and $\varphi_{\mathrm{c} 2}$ are different for different scenarios in Fig. 3, which are given respectively form (11) to (16). 


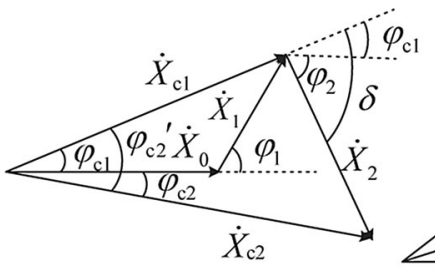

(a)

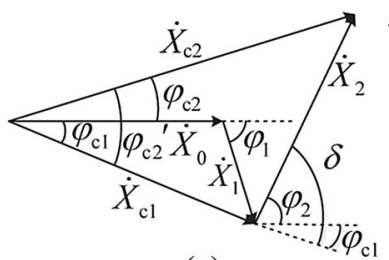

(c)

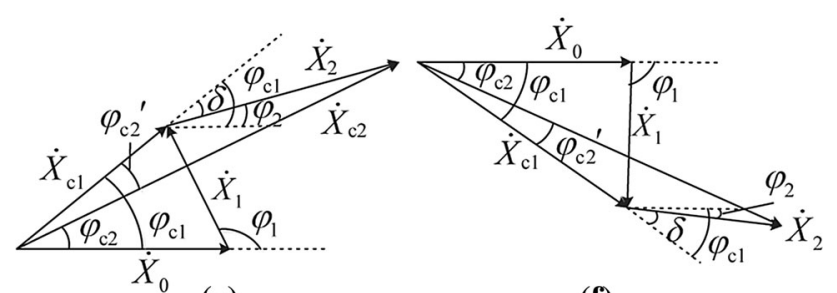

(e)
Fig. 3 Different scenarios for superimposing of $\dot{X}_{0}, \dot{X}_{1}$ and $\dot{X}_{2}$

$$
\begin{aligned}
& \left\{\begin{array}{l}
\delta=\varphi_{2}+\varphi_{\mathrm{c} 1} \\
\varphi_{\mathrm{c} 2}=\varphi_{\mathrm{c} 1}-\varphi_{\mathrm{c} 2}^{\prime}
\end{array}\right. \\
& \left\{\begin{array}{l}
\delta=\varphi_{2}-\varphi_{\mathrm{c} 1} \\
\varphi_{\mathrm{c} 2}=\varphi_{\mathrm{c} 2}^{\prime}+\varphi_{\mathrm{c} 1}
\end{array}\right. \\
& \left\{\begin{array}{l}
\delta=\varphi_{2}+\varphi_{\mathrm{c} 1} \\
\varphi_{\mathrm{c} 2}=\varphi_{\mathrm{c} 2}^{\prime}-\varphi_{\mathrm{c} 1}
\end{array}\right. \\
& \left\{\begin{array}{l}
\delta=\varphi_{2}-\varphi_{\mathrm{c} 1} \\
\varphi_{\mathrm{c} 2}=\varphi_{\mathrm{c} 1}-\varphi_{\mathrm{c} 2}^{\prime}
\end{array}\right. \\
& \left\{\begin{array}{l}
\delta=\varphi_{\mathrm{c} 1}-\varphi_{2} \\
\varphi_{\mathrm{c} 2}=\varphi_{\mathrm{c} 2}^{\prime}+\varphi_{\mathrm{c} 1}
\end{array}\right. \\
& \left\{\begin{array}{l}
\delta=\varphi_{\mathrm{c} 1}-\varphi_{2} \\
\varphi_{\mathrm{c} 2}=\varphi_{\mathrm{c} 2}^{\prime}-\varphi_{\mathrm{c} 1}
\end{array}\right.
\end{aligned}
$$

Now the phase angle of the phasor is considered to be positive, when the phasor advances $\dot{X}_{0}$. The phase angle of the phasor is considered to be negative when the phasor lags $\dot{X}_{0}$. Therefore, for (11), $\dot{X}_{\mathrm{c} 1}$ advances $\dot{X}_{0}$ and $\dot{X}_{2}$ lags $\dot{X}_{0}$. Therefore, $\varphi_{2}$ and $\delta$ are negative, $\varphi_{\mathrm{c} 1}$ is positive. $\delta$ can be gotten by (17). Then, $\varphi_{\mathrm{c} 2}^{\prime}$ computed by (10) is negative, because $X_{\mathrm{m} 2} \sin \delta /\left(X_{\mathrm{mc} 1}+X_{\mathrm{m} 2} \cos \delta\right)$ is negative. $\varphi_{\mathrm{c} 2}$ can be obtained by (18). For (13), $\dot{X}_{\mathrm{c} 1}$ lags $\dot{X}_{0}$ and $\dot{X}_{2}$ advances $\dot{X}_{0}$. Therefore, $\varphi_{2}$ and $\delta$ are positive, $\varphi_{\mathrm{c} 1}$ is negative. $\delta$ can be gotten by (17) as well. Then, $\varphi_{\mathrm{c} 2}^{\prime}$ computed by (10) is positive, because $X_{\mathrm{m} 2} \sin \delta /\left(X_{\mathrm{mc} 1}+X_{\mathrm{m} 2} \cos \delta\right)$ is positive. $\varphi_{\mathrm{c} 2}$ can be obtained by (18). It can be verified that formulas of $\delta$ and $\varphi_{\mathrm{c} 2}$ are the same as (17) and (18) for other scenarios. The detail process is not outlined here.

$\delta=\varphi_{2}-\varphi_{\mathrm{c} 1}$

$\varphi_{\mathrm{c} 2}=\varphi_{\mathrm{c} 2}^{\prime}+\varphi_{\mathrm{c} 1}$

Consequently, $\dot{X}_{\mathrm{c} 2}$ is expressed as:

$$
\begin{aligned}
\dot{X}_{\mathrm{c} 2} & =\sqrt{X_{\mathrm{mc} 1}^{2}+X_{\mathrm{m} 2}^{2}-2 X_{\mathrm{mc} 1} X_{\mathrm{m} 2} \cos \left(\pi-\varphi_{2}+\varphi_{\mathrm{c} 1}\right)} \\
& \angle\left(\varphi_{\mathrm{c} 1}+\arctan \left(\frac{X_{\mathrm{m} 2} \sin \left(\varphi_{2}-\varphi_{\mathrm{c} 1}\right)}{X_{\mathrm{mc} 1}+X_{\mathrm{m} 2} \cos \left(\varphi_{2}-\varphi_{\mathrm{c} 1}\right)}\right)\right) \\
& =\sqrt{X_{\mathrm{mc} 1}^{2}+X_{\mathrm{m} 2}^{2}-2 X_{\mathrm{mc} 1} X_{\mathrm{m} 2} \cos \left(\pi-2 \pi \Delta f_{2} t+\varphi_{\mathrm{c} 1}\right)} \\
& \angle\left(2 \pi \Delta f_{1} t+\arctan \left(\frac{X_{\mathrm{m} 2} \sin \left(2 \pi \Delta \mathrm{f}_{2} \mathrm{t}-\varphi_{\mathrm{c} 1}\right)}{X_{\mathrm{mc} 1}+X_{\mathrm{m} 2} \cos \left(2 \pi \Delta f_{2} t-\varphi_{\mathrm{c} 1}\right)}\right)\right)
\end{aligned}
$$

Figure 4 depicts the magnitude and the phase angle of the superimposition of the rated component, $10 \%$ harmonic of $20 \mathrm{~Hz}$ and $20 \%$ harmonic of $70 \mathrm{~Hz}$. It can be stated that the major frequency components are 20 and $30 \mathrm{~Hz}$. The 20 $\mathrm{Hz}$ component is introduced by the harmonic of $70 \mathrm{~Hz}$, while the $30 \mathrm{~Hz}$ component is introduced by the harmonic of $20 \mathrm{~Hz}$. This can be distinguished by their respective magnitude. In particular, when the $f_{1}$ and $f_{2}$ are symmetric with $f_{0}, \varphi_{10}$ and $\varphi_{20}$ are symmetric with $\varphi_{0}$, and $X_{\mathrm{m} 1}=X_{\mathrm{m} 2}$, $\dot{X}_{\mathrm{c} 2}$ will be magnitude modulated only.

More generally, if more than two harmonics exit in the input signal, the mechanism of frequency transmutation between the harmonic and the phasor oscillation frequencies is the same as that illustrated by Fig. 2 .

The PMU is designed to measure the fundamental component rather than inter-harmonics. But if the interharmonics are not eliminated, their existence results in the fundamental component modulating at the subsynchronous frequency. There are two strategies to dispose of the inter-

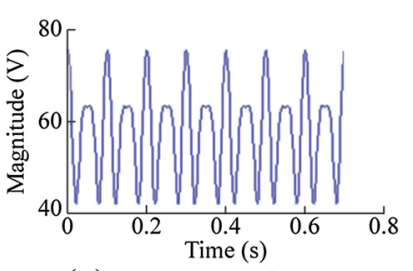

(a) Magnitude of phasor

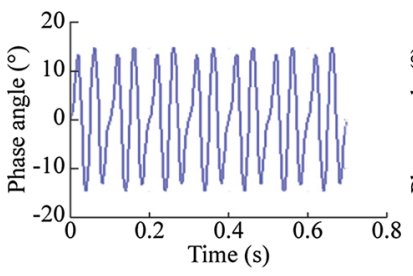

(c) Phase angle of phasor

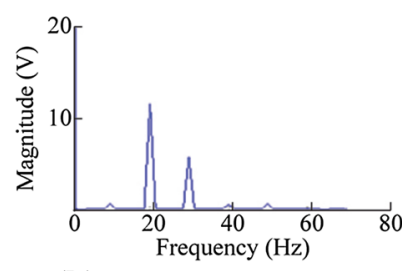

(b) Spectrum of magnitude

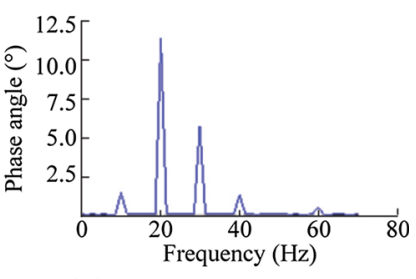

(d) Spectrum of phase angle
Fig. 4 Superimposed phasor with two harmonics 
harmonics. The first one is to remove all of them by filters, and calculate the fundamental phasor only; and the second one is to calculate the superimposed phasor that contains the fundamental component and inter-harmonics. By the second way, we can monitor and detect the subsynchronous oscillation caused by the inter-harmonics with the phasor measurements of PMUs. Then corresponding controls can be carried out to prevent further faults, just like the low frequency oscillation detection based on PMUs.

The PMU measurements are used to analyze the subsynchronous oscillation happed in China in 2015.

\section{Subsynchronous oscillation monitoring based on PMU measurements}

In this section, a subsynchronous oscillation that occurred in western China where there is a large concentration of wind farms and HVDC transmission lines is studied using the recorded PMU data. Results of the analysis show the severe consequences of the inter-harmonics and verify the correctness of the theoretical derivation.

\subsection{Distribution of subsynchronous oscillations}

From July, 2015, several subsynchronous oscillations caused by the inter-harmonics occurred in western China, which resulted in several thermal power generations and the tripping-off of wind farms. PMUs installed in the area tracked the oscillation processes by both recording the signal instant value and measuring the phasor. The total PMU data is over $40 \mathrm{~GB}$ and covers over 140 transmission lines. The subsynchronous oscillations that occurred in September are exemplary, so the PMU data on that day is analyzed.

Figure 5 shows the geographical wiring diagram of the area with the main oscillation components by histograms.
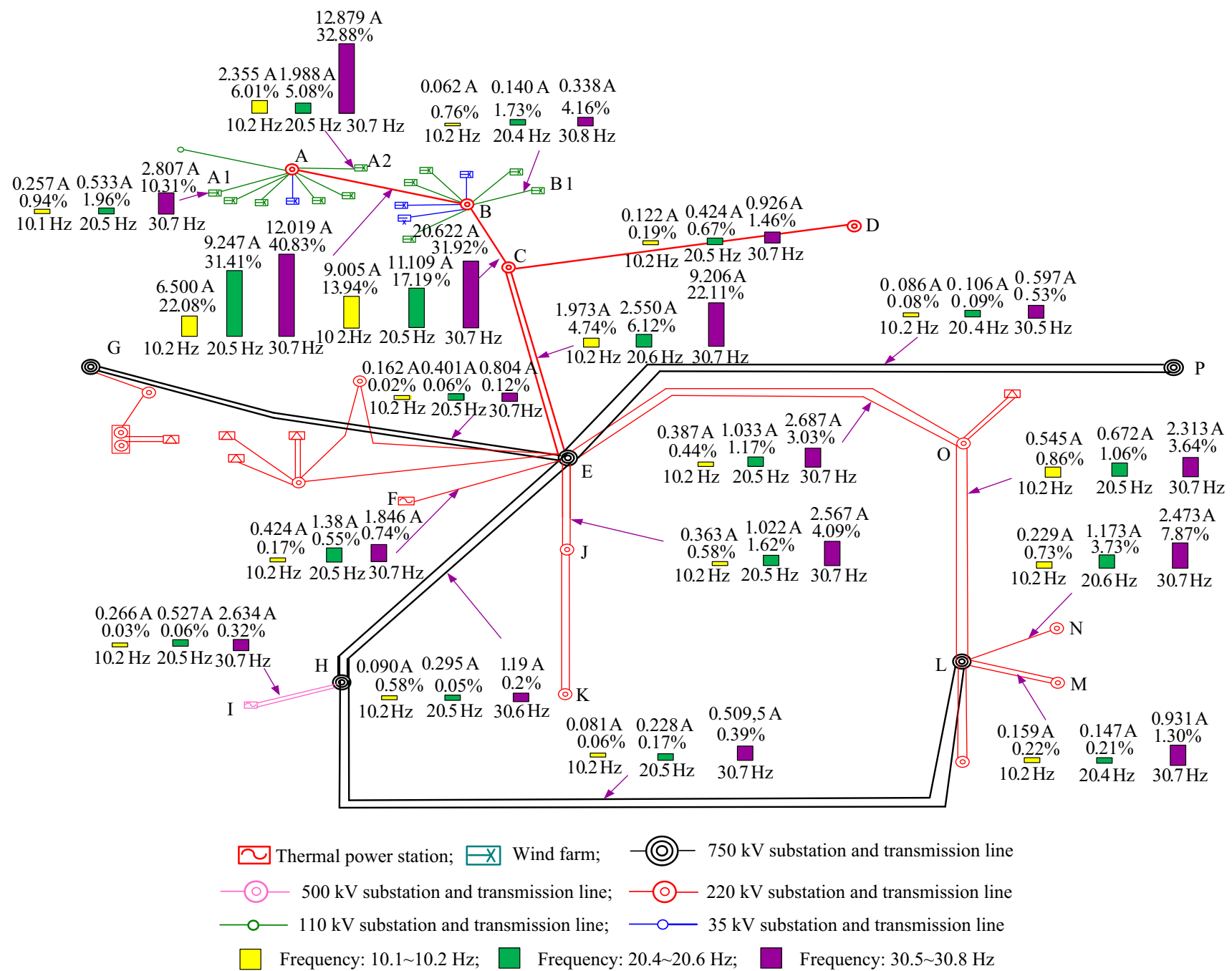

Fig. 5 Oscillation analysis based on PMU measurements 
In the figure, the different colors of the histograms represent different oscillation frequencies. The height of the histograms bars indicates the oscillation magnitudes. The larger the oscillation magnitude is, the higher the histogram bars are. The exact oscillation frequencies are outlined under the histograms, and the oscillation magnitudes and their respective proportions of the fundamental one are outlined above the histograms. It can be seen that all the oscillation frequencies are around 10.2, 20.6, and $30.7 \mathrm{~Hz}$. The highest oscillation magnitude reached $32.88 \%$ of the fundamental component.

The substation A and B are connected with the large scale of wind farms. The substation $\mathrm{H}$ is the convertor station. It can be seen that the oscillation components around wind farms are bigger than those at other places. It means that there are more subsynchronous and supersynchronous harmonics around wind farms. The subsynchronous oscillations are propagated along the transmission lines that cover the voltage levels from 35 to $750 \mathrm{kV}$. The total affected area is over $130000 \mathrm{~km}^{2}$. Farther away and at higher voltage levels, the subsynchronous oscillation magnitudes become smaller. However, the especially high magnitude of the subsynchronous oscillations around the wind farms does not mean that it is the wind farms that cause the inter-harmonics and the subsynchronous oscillation. The exact reason is complicated, and further studies are necessary.

Consequently, the large scale of renewable energy and HVDC introduce a large number of inter-harmonics. They result in the subsynchronous oscillation which can be propagated widely and threaten the security of the power system.

\subsection{Inter-harmonics analysis}

In addition of phasor the measurements, PMUs also recorded the instant values of the signals for some transmission lines. Figure 6 shows the spectrum analysis of the current instantaneous values of the transmission line C-E, while Fig. 7 shows the spectrum analysis of current phasors measurements from the PMU of the same transmission during the same period. To show the inter-harmonics and their impacts on the phasor measurements clearly, the fundamental component is not plotted in Fig. 6 and Fig. 7.

Then, all the frequencies that cannot be ignored appeared in Fig. 6 and Fig. 7 are shown in Fig. 8 in two rows. According to the mechanism of frequency transmutation between the harmonic and the phasor oscillation illustrated by Fig. 2, all the oscillation components can be explained by the corresponding harmonics. For example, the phasor oscillation component at $10 \mathrm{~Hz}$ is caused by the inter-harmonics of 40 and $60 \mathrm{~Hz}$, because their differences

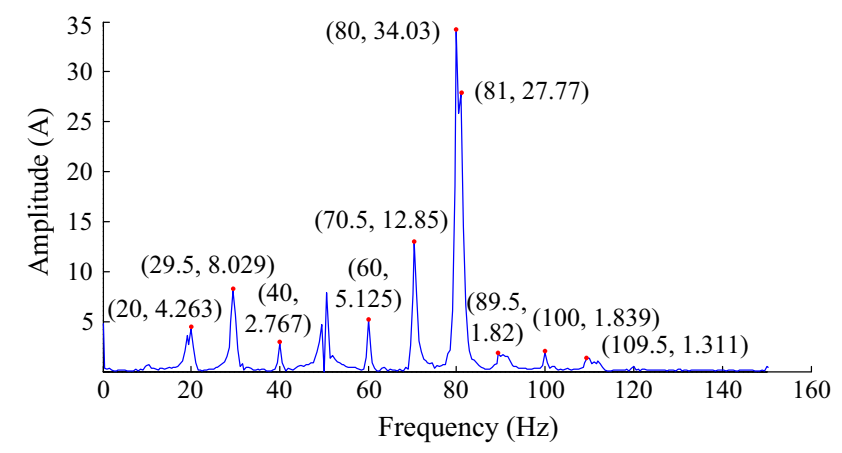

Fig. 6 Spectrum analysis of current instant values of transmission line $\mathrm{C}-\mathrm{E}$

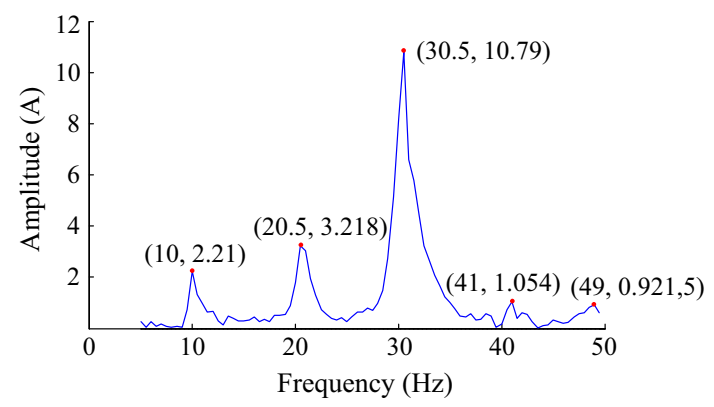

Fig. 7 Spectrum analysis of current phasor of transmission line C-E

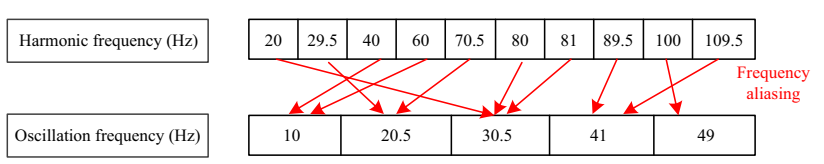

Fig. 8 Corresponding relation between harmonic and oscillation frequencies

between the rated frequency are both $10 \mathrm{~Hz}$. As revealed in Section 3, one oscillation component can be caused by multiple subsynchronous or supersynchronous harmonics. Therefore, the derivations in Sections 2 and 3 are verified by the real PMU measurements and recorded data. Since it is feasible to identify the oscillation from the active power, Fig. 9 depicts the spectrum analysis of the active power of the same line during the time window measured by PMUs. It can be seen that the oscillation frequencies are the same as that in Fig. 7.

It may seem that the $109.5 \mathrm{~Hz}$ harmonic appears to be an exception. According to the mechanism, such a harmonic should result in phasor oscillation at $59.5 \mathrm{~Hz}$. But, the highest reporting rate of PMUs in China is $100 \mathrm{~Hz}$, which cannot meet the Shannon sampling theorem. Therefore, the frequency aliasing occurs, the phasor oscillation frequency become $40.5 \mathrm{~Hz}$. Since the frequency aliasing is 


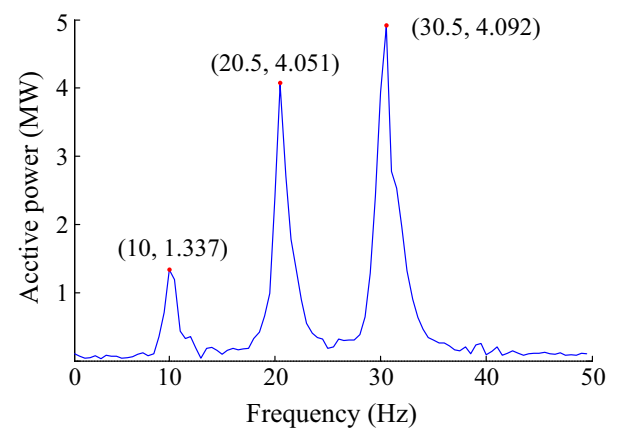

Fig. 9 Spectrum analysis of active power of transmission line C-E

irreversible, it will be considered that there is more harmonic of $109.5 \mathrm{~Hz}$ than there should be.

Consequently, when the frequency aliasing occurs, phasor measurements cannot restore the original harmonics accurately. Measures should be taken to prevent the frequency aliasing from occurring, especially in the case of the subsynchronous oscillation. For example, a digital filter in the PMU algorithm can eliminate the oscillation components which may lead to the frequency aliasing.

As shown in Fig. 8, one subsynchronous oscillation frequency component of the phasors may be caused by two harmonics that are symmetrical about the rated frequency. Based on nowadays PMU, we can only tell if there are subsynchronous oscillation that maybe caused by the subsynchronous or supersynchronous inter-harmonics. But the exact magnitude of the subsynchronous and supersynchronous inter-harmonics cannot be obtained. Further algorithms for the inter-harmonic monitoring based on PMUs are needed to achieve that. In addition, the fast changing phasor makes precise measurement more difficult. Because, DFT assume that the phasor is static in the data window. The faster the phasor changes, the bigger the measurement error is [17]. Therefore, relevant phasor measurement algorithms which can track the phasor modulated in subsynchronous frequency precisely are also needed.

\section{Conclusion}

In this paper, the impacts of inter-harmonics on synchrophasor measurements are revealed. The phasor expression of a signal that contains the fundamental component and single or multiple inter-harmonics is proposed. It is found that the harmonics can cause the subsynchronous oscillation of the phasor measurements. The frequency of the oscillation of phasor measurements is the difference between the harmonic and the fundamental component.
The instantaneous value of the power signal and the phasor measurements obtained by PMUs during a subsynchronous oscillation from a real power grid that has a large concentration of wind farms and HVDC transmission lines are analyzed. The geographical wiring diagram with the supersynchronous oscillation distribution shows that more inter-harmonics are located near the wind farms, and many voltage levels and a wide area are impacted. Furthermore, the spectrum analysis comparison between the instantaneous value of the power signal and the phasor demonstrate the correctness of the theoretical derivation.

The monitoring of inter-harmonics using PMUs is possible and necessary. However, one subsynchronous oscillation frequency component of the phasors may be caused by two harmonics, and frequency aliasing may occur. Therefore, how to restore the harmonics precisely based on the phasor measurements of PMUs still need further studies.

Open Access This article is distributed under the terms of the Creative Commons Attribution 4.0 International License (http:// creativecommons.org/licenses/by/4.0/), which permits unrestricted use, distribution, and reproduction in any medium, provided you give appropriate credit to the original author(s) and the source, provide a link to the Creative Commons license, and indicate if changes were made.

\section{References}

[1] Phadke AG (1993) Synchronized phasor measurements in power systems. IEEE Comput Appl Power 6(2):10-15

[2] Phadke AG, Thorp JS (2008) Synchronized phasor measurements and their applications. Springer, Boston, pp 3-5

[3] Zhang YC, Markham P, Xia T et al (2010) Wide-area frequency monitoring network (FNET) architecture and applications. IEEE Trans Smart Grid 1(2):159-167

[4] Bhatt NB (2011) Role of synchrophasor technology in the development of a smarter transmission grid. In: Proceedings of the 2011 IEEE power and energy society general meeting, Detroit, MI, 24-29 Jul 2011, 4 pp

[5] Chen J, Shrestha P (2012) Use of synchronized phasor measurements for dynamic stability monitoring and model validation in ERCOT. In: Proceedings of the 2012 IEEE power and energy society general meeting, San Diego, CA, 22-26 Jul 2012, $7 \mathrm{pp}$

[6] Wu HR, Wang Q, Li XH (2008) PMU-based wide area damping control of power systems. In: Proceedings of the joint international conference on power system technology and IEEE power India conference, New Delhi, 12-15 Oct 2008, 4 pp

[7] Wu M, Xie L, Cheng L et al (2016) A study on the impact of wind farm spatial distribution on power system sub-synchronous oscillations. IEEE Trans Power Syst 31(3):2154-2162

[8] Belkin P (2010) Event of 10-22-09. In: Proceedings of the technical conference: competitive renewable energy zone (CREZ) system design and operation, 26 Jan 2010, Taylor, TX

[9] Wan YH (2013) Synchronized phasor data for analyzing wind power plant dynamic behavior and model validation. NREL/TP5500-57342, National Renewable Energy Laboratory (NREL), Golden, CO 
[10] Wu M, Sun R, Cheng L et al (2014) Parameter sensitivity analysis for sub-sybchronous control interactions in wind-integrated power systems. In: Proceedings of the CIGRE US National Committee 2014 grid of the future symposium, Houston, TX, 19 Oct 2014

[11] IEEE Std C37.118.1-2011. IEEE standard for synchrophasor measurements for power systems (2011)

[12] GB/T 26862-2011. Test specification for synchrophasor measurement unit for power system (2011)

[13] IEEE Std C37.118-2005 (Revision of IEEE Std 1344-1995). IEEE standard for synchrophasors for power systems (2006)

[14] Q/GDW 131-2006. Technology guidance of power system real time dynamic monitoring system. State Grid Corporation of China (SGCC), Beijing (2006)

[15] Liu H, Bi TS, Yang QX (2013) The evaluation of phasor measurement units and their dynamic behavior analysis. IEEE Trans Instrum Meas 62(6):1479-1485

[16] Kang SH, Lee DG, Nam SR et al (2009) Fourier transform-based modified phasor estimation method immune to the effect of the DC offsets. IEEE Trans Power Deliver 24(3):1104-1111

[17] Bi TS, Liu H, Feng Q et al (2015) Dynamic phasor model-based synchrophasor estimation algorithm for M-class PMU. IEEE Trans Power Deliver 30(3):1162-1171

Hao LIU was born in China in 1985. He received his Ph.D degree at North China Electric Power University. He is currently a lecturer at North China Electric Power University. His research interests include synchronized phasor measurement technique and its application.

Tianshu BI received her Ph.D degree at the Department of EEE in the University of Hong Kong in 2002. She is currently a professor at
North China Electric Power University. Her research interests include power system protection and control, synchronized phasor measurement technique and its application and fault diagnose.

Xiqiang CHANG is a senior engineer, his main research interest is power system analysis and control.

Xiaolong GUO is an engineer, his main research interest is power system analysis and control.

Lu WANG was born in China in 1991. She is currently a Master Degree candidate at North China Electric Power University, Beijing, China. Her research interest is PMU testing technology.

Chuang CAO was born in China in 1991. He is currently a Master Degree candidate at North China Electric Power University, Beijing, China. His research interest is PMU measurement quality.

Qing YAN is a senior engineer, his main research interest is power system operation and management.

Jinsong LI was born in China in 1981. He received Master degree at Beijing University of Posted and Telecommunications in 2010. He is currently working at the Power Automation Department of the China Electric Power Research Institute. His research interests include synchronized phasor measurement technique, smart substation automation technologies and its application. 